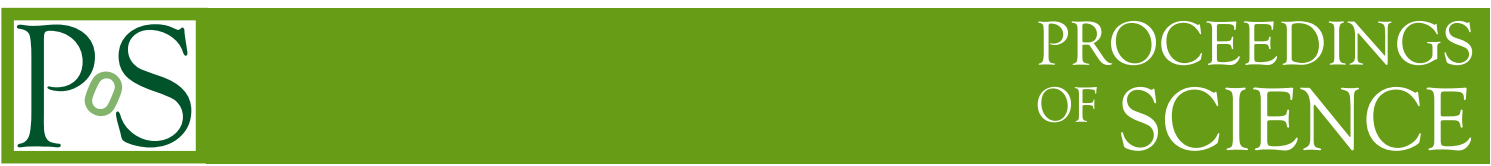

\title{
Charm physics with the CHORUS experiment
}

\section{Giovanni De Lellis*}

Università di Napoli "Federico II", Italy

E-mail: giovanni.de.lellis@cern.ch

High energy neutrino interactions induce charmed hadron production at the level of a few percent and therefore they constitute a powerful tool to study charm physics. The CHORUS experiment can profit of the extremely high spatial resolution of nuclear emulsions to identify and study the charm production and decay vertexes. This experiment is now analyzing the final data sample and publishing new results on the charm physics with the muon neutrino beam at CERN. In this paper we review these results in the general framework of charm physics with neutrinos.

International Europhysics Conference on High Energy Physics

July 21st - 27th 2005

Lisboa, Portugal

*Speaker. 


\section{Introduction}

Neutrino and anti-neutrino induced charm-production is particularly interesting in many respects [1]. So far only two experiments, E531 [2] and CHORUS [3], have studied this process with nuclear emulsions. Owing to their submicrometric position resolution, emulsions allow the charmed particle identifi cation through the visual observation of its decay in an almost background free environment. This turns into a very good sensitivity to the charm production also in the low energy region. Nuclear emulsion technique has recently shown a large revival due to the impressive development in the fi eld of automatic scanning microscopes $[4,5,6]$.

\section{The CHORUS experiment}

Originally designed to search for $v_{\mu} \rightarrow v_{\tau}$ oscillations through the direct observation of the $\tau$ decay, the CHORUS experiment is also studying the production and decays of charmed hadrons.

The event reconstruction is initiated by electronic detectors which predict the particle track position in the emulsions with a resolution of about $200 \mu \mathrm{m}$ in position and $3 \mathrm{mrad}$ in angle. The track is then followed up in the emulsions to the interaction vertex. All the track segments in a volume of about $13 \mathrm{~mm}^{3}$ around the interaction vertex are involved into the offline tracking procedure to reconstruct tracks and vertexes [7].

\section{Charm search in emulsions}

Table 1: CHORUS neutrino induced charm sample.

\begin{tabular}{crr}
\hline Decay prongs & Data sample & Background yield \\
\hline 1 & 452 & $43 \pm 3$ \\
2 & 819 & $36.6 \pm 3.5$ \\
3 & 491 & $3.8 \pm 0.2$ \\
4 & 226 & \\
5 & 22 & $1.5 \pm 0.1$ \\
6 & 3 & \\
\hline Total sample & 2013 & $85 \pm 5$ \\
\hline
\end{tabular}

An event is classifi ed as a charm candidate if there is the evidence for a secondary decay besides the primary vertex. If the decaying particle is neutral, a matching with the electronic detector is also required to insure the time coincidence of the two vertexes. The event analysis is ended by the visual inspection at the microscope. In Table 1 , the number of charm candidates validated by the visual inspection is reported for each decay topology. This sample corresponds to 93,807 charged-current (CC) interactions with a negative muon in the fi nal state.

The background level for the charm search in emulsions is low. Indeed, interactions can be distinguished from decays unless they do not show any fragment or Auger electron from the nuclear break-up (so-called "white" interactions). Their interaction length is by several order of magnitude 
larger than the flight lengths of charmed hadrons. Strange particle decays are also suppressed by the much longer lifetime. The background yield is reported in Table 1 for each decay channels.

\section{1 $D^{0}$ studies}

A study of the $D^{0}$ meson production and decays was performed [8]. After the event selection and eye inspection, 1048 candidates were found, as shown in Table 1. After background subtraction and effi ciency correction the two prong decay branching ratio was found to be $b r_{2}=0.647 \pm$ $0.049 \pm 0.031$. We have also obtained the first measurement of the six prong branching ratio as $b r_{6}=\left(1.2_{-0.9}^{+1.3} \pm 0.2\right) \times 10^{-3}$.

The fully neutral $D^{0}$ topological branching ratio has also been measured. This quantity was previously assumed to be about 5\% [9]. Since this decay mode is not detectable in the experiments recognising the $D^{0}$ through its charged daughters, the measurement affects many of the results published in the past by other experiments.

The fully neutral branching ratio can be written as:

$$
b r_{0}=1-b r_{4}\left(1+\frac{b r_{2}}{b r_{4}}+\frac{b r_{6}}{b r_{4}}\right)
$$

where $b r_{4}$ has been precisely measured to be $(13.38 \pm 0.58) \%$ through the measurement of the particle width ratio, $\Gamma_{4} / \Gamma_{\text {total }}$ [9]. Therefore we got $b r_{0}=0.218 \pm 0.049 \pm 0.036$.

The cross-section measurement relative to the $\mathrm{CC}$ one gives

$$
\frac{\sigma_{D^{0}}}{\sigma_{C C}}=0.0269 \pm 0.0018 \pm 0.0013
$$

\section{2 $D^{*}$ production}

The identification of the $D^{*+}$ in this experiment is based on the $D^{*+} \rightarrow D^{0} \pi^{+}$decay detection [10]. This decay is characterised by low transverse momentum $\left(p_{\perp} \leq 39 \mathrm{MeV} / c\right)$ and, therefore, the $\pi^{+}$has a low momentum $(\leq 4 \mathrm{GeV} / c)$ and small angle with respect to the $D^{0}$. In emulsions this event is seen as a $D^{0}$ decay with a positive hadron at the primary vertex emitted with a small $p_{\perp}$ with respect to the $D^{0}$.

The momentum of the hadrons is measured by the hadron spectrometer downstream of the target. 488 visually inspected $D^{0}$ 's with a flight length larger than $100 \mu \mathrm{m}$ are used as a starting sample. 377 hadron tracks at the primary vertex have the momentum measured. The hadron is required to be positive and its angle with respect to the $D^{0}$ to be less than $60 \mathrm{mrad}$. Negative hadrons are used for the background estimation.

After background subtraction, the $p_{\perp}$ distribution for positive hadrons shows an excess of $22.1 \pm 5.5$ events in the range 10 to $50 \mathrm{MeV} / c$ which can be attributed to $D^{*+}$ decays. The ratio of cross-sections $\sigma\left(D^{*}\right) / \sigma\left(D^{0}\right)=0.38 \pm 0.09 \pm 0.05$ is measured. Given the $D^{0}$ cross-section measurement [8], the $D^{*}$ production rate relative to $\mathrm{CC}$ events is

$$
\frac{\sigma\left(D^{*}\right)}{\sigma_{C C}}=[1.02 \pm 0.25 \pm 0.15] \% .
$$




\section{$3.3 \bar{v}_{\mu}$ induced charm production}

Charm production in $\bar{v}_{\mu} \mathrm{CC}$ interactions comes mainly (about $90 \%$ ) from a scattering off a ${ }^{-} s$ sea quark. Therefore, the combined analysis with neutrino charm production can provide a good separation between valence and sea contributions. Moreover, unlike the neutrino case, the quasielastic production is forbidden and $\bar{\Lambda}_{c}$ formation is suppressed by the baryon number conservation.

A data sample made of 2704 interactions with a positive spectrometer muon in the fi nal state has been analysed [11]. 32 charm candidates have been confi rmed by the visual inspection with an estimated background of $3.2 \pm 04$. The cross-section measurement for this process gives

$$
\frac{\sigma\left(\bar{v}_{\mu} N \rightarrow \mu^{+-} c X\right)}{\sigma\left(\bar{v}_{\mu} N \rightarrow \mu^{+} X\right)}=\left(5.0_{-0.9}^{+1.4} \pm 0.7\right) \%
$$

which has to be compared with roughly the $6.0 \%$ observed in neutrinos $[8,12]$. This difference is consistent with the softer $\bar{v}_{\mu}$ energy spectrum and with the absence of the quasi-elastic process.

The ratio between neutral and charged charmed particles is

$$
\frac{f_{\bar{C}^{0}}}{f_{C^{-}}}=2.6_{-1.0}^{+1.7} \pm 0.8
$$

to be compared with the $0.97 \pm 0.15 \%$ measured with neutrinos, showing the expected suppression of $\bar{\Lambda}_{c}$ production.

\subsection{Associated charm production}

A gluon emitted via bremsstrahlung converting into a charm quark pair produces two charmed hadrons both in charged- and in neutral-current interactions. In the latter case, the $Z^{0}$-gluon fusion mechanism has to be added. These processes are very rare and there is neither a well-established measurement nor a good theoretical prediction, especially for CC events. CHORUS has published the first observation of one event with associated charm production in CC interaction [13].

\section{References}

[1] G. De Lellis, P. Migliozzi and P. Santorelli, Phys. Rept. 399, 227 (2004).

[2] Ushida et al., Phys. Lett. B206 (1988) 375.

[3] CHORUS Coll., Nucl. Instr. Meth. A401 (1997) 7.

[4] S. Aoki et al., Nucl. Instr. Meth. B51 (1990) 466.

[5] N. Armenise et al., Nucl. Instrum. Meth. A551 (2005) 261.

[6] M. De Serio et al., Nucl. Instrum. Meth. A554 (2005) 247.

[7] K. Kodama et al., Nucl. Instr. Meth. A493 (2002) 45.

[8] CHORUS Coll., Phys. Lett. B613 (2005) 105.

[9] S. Eidelman et al. [Particle Data Group], Phys. Lett. B 592, 1 (2004).

[10] CHORUS Coll., Phys. Lett. B614 (2005) 155.

[11] CHORUS Coll., Phys. Lett. B 604, 11 (2004).

[12] CHORUS Coll., Phys. Lett. B555 (2003) 156.

[13] CHORUS Coll., Phys. Lett. B539 (2002) 188. 